American Journal of Applied Sciences 8 (5): 463-467, 2011

ISSN 1546-9239

(C) 2010 Science Publications

\title{
Use of Optical Reflectometry to Observe Various Concentrations of Glucose
}

\author{
Hariyadi Soetedjo \\ Center for Integrated Research and Innovation (CIRNOV) and Study Program of Physics, \\ The University of Ahmad Dahlan, Jl. Cendana No 9A, \\ Yogyakarta 55166, Indonesia
}

\begin{abstract}
Problem statement: Monitoring technique on glucose is a hot topic as this compound has been considered to be important in human healthties. Optical reflectometry has been used to study various concentrations of glucose dissolved in the water. Approach: The self-construction of reflectometer used was of hemi-cylinder aluminum to allow light beam strikes the sample at $70^{\circ}$ for sor p-polarization state. The cell for sample was of a hemisphere cavity introduced in the hemi-cylinder face down the surface of stage. The visible light was used as a light source, meanwhile dark Nilon material for a stage has been used to allow the specular reflection by the interface introduced between the liquid sample and the stage. Results: From the investigation, the change of concentrations could be observed from the sensitive change of reflected intensities as indicated by a shift of peak wavelength. Conclusion: The measurement results obtained from a simple set up was encouraging for glucose concentration monitoring for wide applications.
\end{abstract}

Key words: Optical reflectometry, glucose concentration, reflected intensities, p-polarizations, various concentrations, Surface Plasmon Resonance (SPR), Reflectometric Interference Spectroscopy (RIfS), DNA molecules, optical heterodyne, hemi-cylinder

\section{INTRODUCTION}

Measurement technique based on optical reflection is considerably interesting as many applications such of biosensors for molecules detection have been used widely. Use of this technique shows a great interest particularly in hybridization process of DNA molecules for DNA sensor. This technique shows many practical applications for forensics, identifications of pathogenic species and DNA damage by chemical and pollutants. For biosensors, a label-free measurement using optical techniques such use of Surface Plasmon Resonance (SPR) and Reflectometric Interference Spectroscopy (RIfS) (Sauer et al., 1999; Birket et al., 2002) is more interesting compare than that of using fluorescent treatment because this technique introduces many procedures leads to long process and error in analysis. In health care applications, glucose is one important compound that has been studied its characteristics (Lee et al., 2004; Jiang et al., 2003). Many research works were carried out to monitor glucose content in a human body as this study introduces an important problem in medical applications (Harrison et al., 2009). Any development in detection technique for glucose monitoring based on optics were performed such use of an optical heterodyne polarimeter for in vivo noninvasive monitoring (Chou et al., 1998), use of vertical cavity semiconductor lasers for glucose absorption measurement (Fards et al., 2007), noninvasive monitoring using an optical coherence tomography (Esenaliev et al., 2001). It has been shown that in the measurement using optical reflection property, the sensitivity of measurement could be improved by introducing s-or p-polarization to the incident light and then analyzing further reflection properties. This strategy leads the study of different phase experienced by light propagation through multilayer media. This method could offer more interesting strategy for layer characterizations. By referring to that advantage, some apparatuses have been built up such of optical reflectometer that introducing simple in design, operation and low cost and capable to find out the absolute values of optical properties of thin film. A reflectometer introduced with a fixed angle of polarizer at s-or p-state polarization $\left(0-90^{\circ}\right)$ has been succeeded to monitor thin layer presence on the surface of probe window due to a contact between lignin liquid (Soetedjo and Ratty, 2003). This technique is based on a polarized incident light beam guided through a fiber optic directed onto the sample and reflected light intensities were recorded by a spectrometer. Measurement was carried out by varying incident angles for a single wavelength. From the result, it was found that the absolute optical layer thickness and refractive indexes were determined. This technique is considerably simple in operation that has been used to 
characterize organic ultra thin film deposited on the silicon wafer (Hariyadi et al., 1999). In this study, we report the observation of various glucose concentrations by using an optical reflectometer. The measurement has been carried out by introducing s- and p-polarizations of incident beam before strikes the sample for an incident angle of $70^{\circ}$. The reflected intensities off the sample recorded by a spectrometer have been used for further analysis. Measurement was done at room temperature using a simple self-construction apparatus and low cost.

\section{MATERIALS AND MATHODS}

For light propagation through a multilayer media system, light will experience any reflection and refraction due to the interface exists between the different media. Figure 1 shows the schematic diagram for the above phenomena for a certain incident angle, $\theta$. From that figure, the incident light strikes the interface layer that is introduced between medium $\mathrm{m}$ and $\mathrm{L}$. Reflectance of the multilayer media, $\mathrm{R}$ will be recorded by the spectrometer, detector for further analysis in the measurement. The spectral intensities obtained were subtracted to the background intensities. The reflectance could be obtained as a square of multiple reflections, $\mathrm{r}$ by referring to the common formula derived from the Maxwell's equation below (Bass, 1994). Detail equation could be found in Soetedjo and Ratty (2003).

$$
\begin{aligned}
& r=\frac{\eta_{m} E_{m}-H_{m}}{\eta_{m} E_{m}+H_{m}} \\
& R=r \cdot r
\end{aligned}
$$

Where

$\mathrm{r}^{*} \quad=$ Complex conjugate of $\mathrm{r}$

$\mathrm{m}=\mathrm{m}$-th layer of incident medium $(\mathrm{m}=0$ is for a substrate)

$\mathrm{E}_{\mathrm{m}}, \mathrm{H}_{\mathrm{m}}=$ Electric and magnetic vectors respectively, in the incident medium

$\mathrm{n}=\left\{\begin{array}{ll}\frac{\mathrm{n}}{\cos \theta_{\mathrm{o}}} & \mathrm{p}-\text { polarization } \\ \mathrm{n} \cos \theta_{0} & \mathrm{p}-\text { polarization }\end{array}\right\}$

where, $\mathrm{n}$ is Refractive index of each layer and $\theta_{0}$ is an angle of incident.

A self-construction of apparatus used in this measurement is shown schematically in Fig. 2. The cell for sample was prepared by introducing a cavity of a hemi-sphere (about $3 \mathrm{~mm}$ in diameter) in the hemi-cylinder aluminum faces down to the surface of dark Nilon material. The hollows (1 $\mathrm{mm}$ in diameter) provided in the aluminum are fixed at a certain angle of $70^{\circ}$ for incidence and specular reflected light path to allow the beam strikes the sample and then recorded by a spectrometer. This incident angle is chosen because the Brewster angle for this sample medium was assumed to experience around that angle as observed by many researchers in biosensors (Soetedjo et al., 2010). This selection of angle is done by a consideration that sensitivity of measurement could be improved particularly at s-polarization. Light source of a $35 \mathrm{~W}$ white lamp was used to illuminate the sample through a hollow provided by a fiber optic. A polarizer was placed after the light source that could be adjusted at s- or p-polarization state before strike the sample. Later on, light beam reflected off the sample will be recorded by a spectrometer (Ocean Optics) for data collection. The set up has been used successfully for investigating the optical reflection of nitrocellulose membrane (Soetedjo, 2011). The measurement was carried out to various concentrations of glucose (Merck) from 0.0-0.5 g dissolved in $25 \mathrm{~mL}$ pure water, respectively (notation of $25 \mathrm{~mL}$ solvent will not be printed in the next sentences). The solution was dropped into the cell through a vertical inlet down to the cavity. After the sample (glucose solution) measured, then it was blown out using air to drain out the liquid before run another sample for subsequent measurement.

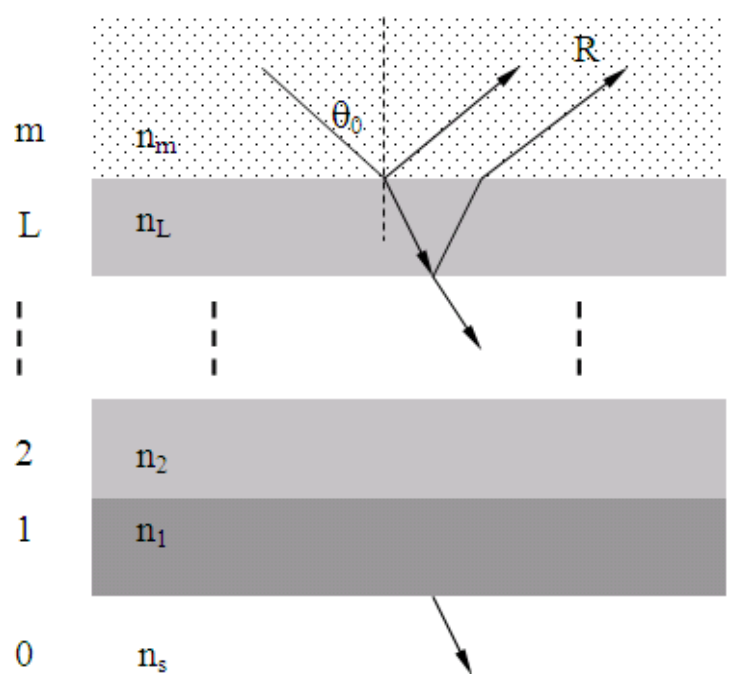

Fig. 1: A schematized diagram of light propagation through multilayer system 
Am. J. Applied Sci., 8 (5): 463-467, 2011

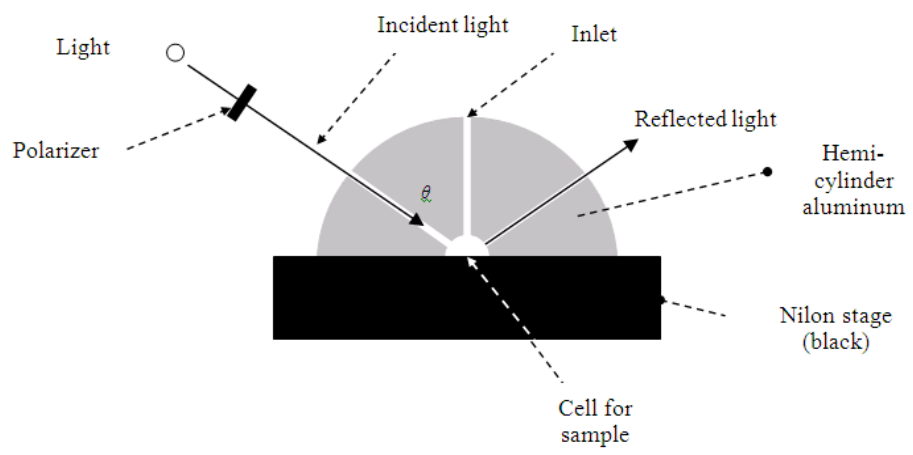

Fig. 2: Schematized diagram of a polarized reflectometer used

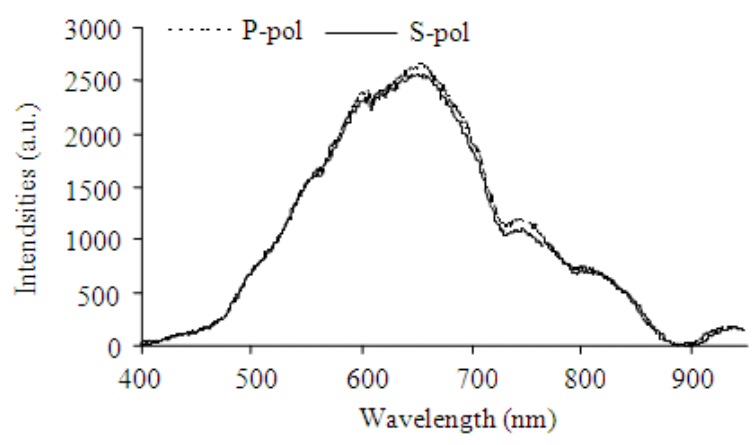

Fig. 3: Spectroscopic intensities recorded by spectrometer for incident light at s- and ppolarizations

The nilon stage used is able to introduce reflected light experienced by the interface between the medium of sample and the stage.

\section{RESULTS}

Experimental results have been obtained for initial measurement of light intensities recorded at incident angle as given in Fig. 3 for s-and p-polarization states. Spectral curve at p-polarization shows a bit higher than that of the s-polarization particularly from $600-800 \mathrm{~nm}$. Measurements were done to various concentrations of glucose as reflected intensities obtained for s-and ppolarizations (guided by a dashed-arrow) shown in Fig. 4 $(a, b)$, respectively. From that figure, the reflected intensities for pure water $(0 \mathrm{~g})$ are relatively highest among the other spectral intensities of glucose content.

By observing the peaks occurs for various concentration of glucose, the interesting phenomenon was found as the increase of concentration up to $0.4 \mathrm{~g}$ will be followed by the significant shift of peak wavelength to a greater value as shown in Fig. 5 for both polarizations. These curves are deduced from the results plotted in Fig. 4.

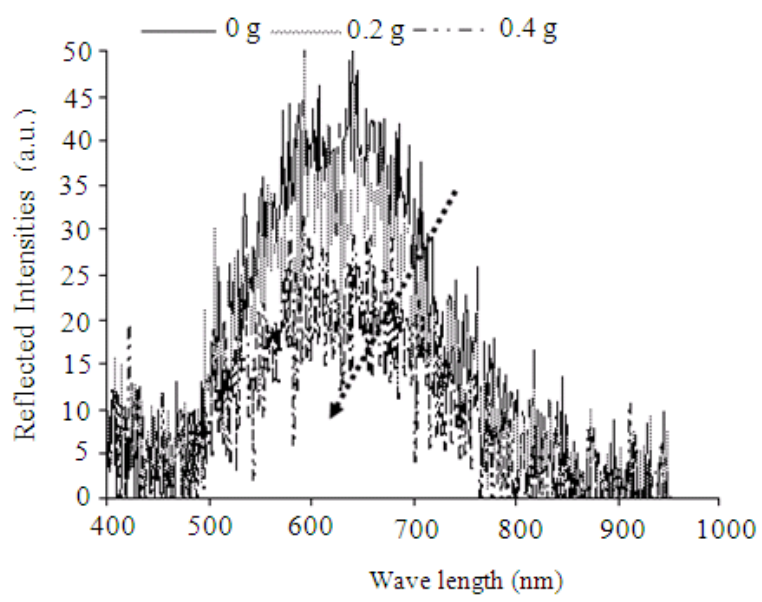

(a)

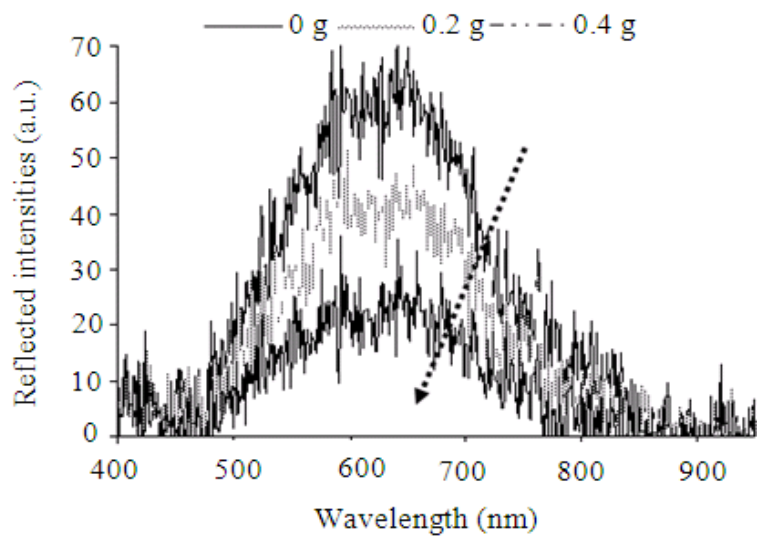

(b)

Fig. 4: Reflected intensities of various concentrations of glucose dissolved in $25 \mathrm{~mL}$ pure water for (a) s- and (b) p-polarization, respectively

Later on, the curve is found to be stable from 0.4-0.5 g. The reflected intensities for s-and p-polarizations $\left(R_{s}\right.$ and $R_{p}$, respectively) sat a wavelength of $580 \mathrm{~nm}$ have been observed as shown in Fig. 6. 


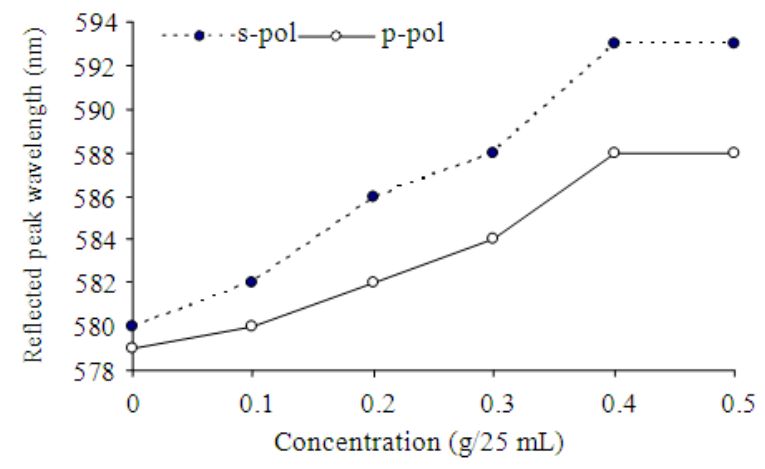

Fig. 5: Relationship between various concentrations of glucose and peak wavelength of reflected intensities observed at (a) s-polarization and (b) p-polarization

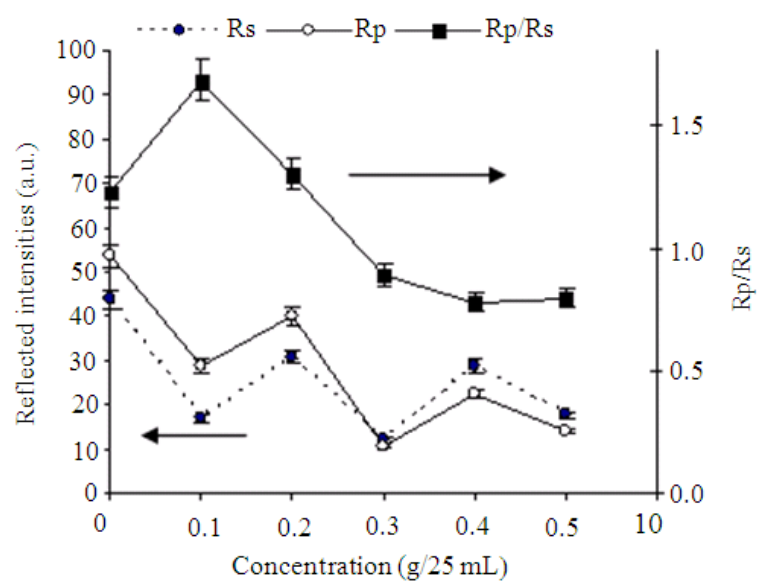

Fig. 6: Relationship between various concentrations of glucose and reflected intensities observed at $580 \mathrm{~nm}$

\section{DISCUSSION}

As we can see in Fig. 3, a bit higher spectral curve for p-polarization is due to the phenomenon of light wave that propagates parallel (p-polarization) contribute much intensities rather than for spolarization that propagates perpendicularly (spolarization) respect to the wave plane. Meanwhile, major peaks are observed at around $630 \mathrm{~nm}$ for both states of polarization followed by other minor peaks.

Figure 4 shows the intensities for all spectra observed are also higher for p-polarization. Due to scattering effect, the reflected intensities recorded by the spectrometer are fluctuating. The increase of glucose concentration is followed by the decrease of the reflected intensities. However, the increase from 0.3-0.5 $\mathrm{g}$ is found to have constant amplitudes as the curves overlap each other (not shown in the figure). By comparing of all spectral intensities, there are weak peaks arise at a wavelength around 600 and $650 \mathrm{~nm}$ for both polarizations, as the peaks display is not clear due to the scattering effect.

For another phenomenon as we can see in Fig. 5, the wavelength of s-polarization is found to be higher than that of p-polarization. This phenomenon may be due to the property of light wave of s-polarization that propagates perpendicularly respects to the wave plane that could introduce relatively higher reflection.

Figure 6 shows that the curves are oscillating for both polarizations and trend to decrease by the increase of the concentration. The ratio between those reflected intensities $\left(R=R_{p} / R_{s}\right)$ decreases moderately before going stable for concentration starting from 0.4-0.5 g. The intensities are increased from the initial concentration of no glucose content (pure water). The observation was not carried out to the concentration of glucose greater than $0.5 \mathrm{~g}$.

\section{CONCLUSION}

From the measurement done to various concentrations of glucose using a self-construction of reflect meter, the increase of concentration from $0-0.5 \mathrm{~g}$ (dissolved in $25 \mathrm{~mL}$ pure water) could be observed through a shift of peak wavelength to the greater wavelength in visible light spectrum. The curve decreases moderately by the increase of concentration up to $0.4 \mathrm{~g}$ and then going stable up to $0.5 \mathrm{~g}$ for both polarizations (s-and p-polarizations). The set-up apparatus used is simple, low cost and shows encouraging study of the glucose concentration based on the optical reflection.

\section{ACKNOWLEDGEMENT}

The researcher would like to thank to the Laboratory of Biology, FMIPA, The University of Ahmad Dahlan, Yogyakarta, Indonesia for the chemicals used in this research study.

\section{REFERENCES}

Bass, M., 1994. Handbook of Optics. 2nd Edn., McGraw-Hill, Inc., New York, ISBN: 10: 007047740X, pp: 1664.

Birket, O., HM. Haake, A. Schütz, J. Mach and A. Brecht et al., 2002. A streptavidin surface on planar glass susbtrates for the detection of biomolecular interaction, Anal. Biol., 282: 200-208. DOI: $10.1006 /$ abio.2000.4615 
Chou, C., C.Y. Han, W.C. Kuo, Y.C. Huang and C.M. Feng, 1998. Noninvasive glucose monitoring in vivo with an optical heterodyne polarimeter, Applied Optics, 37: 3553-3557. DOI: 10.1364/AO.37.003553

Esenaliev, E.O., K.V. Larin and I.V. Larina, 2001. Noninvasive monitoring of glucose concentration with optical coherence tomography. Optics Lett., 26: 992-993. DOI: 10.1364/OL.26.000992

Fards, S.T., W. Hofmann, P.T. Fard, G. Böhm and M. Ortsiefer et al., 2007. Optical absoprtion glucose measurements using $2.3 \mu \mathrm{m}$ vertical cavity semiconductor lasers. IEEE Photonics Tech., Lett., 1: $1-3$.

Hariyadi, D.R. Lovet and S.R.P Smith, 1999. Optical ellipsometry (polarization) measurements on alkyl ammonium metal complexes, J. Mater. Sci., Lett., 18: 1037-1038. DOI: 10.1023/A:1006675526758

Harrison, A.P., R.G. Cooper, M.A. Suliman and U. AlAlami, 2009. The Efficacy of Karkadeh Tea in Controlling Post-Prandial Blood Glucose Levels. Am. J. Pharmacol. Toxicol., 4: 151-157. DOI: 10.3844/ajptsp.2009.151.157

Jiang, D., E. Liu, X. Chen and J. Huang, 2003. Design and properties study of fiber optic glucose biosensor. Chinese Optics Lett., 1: 108-110.
Lee, Y.J., S.A. Pruzinsky and P.V. Braun, 2004. Glucose-sensitive inverse opal hydrogels: Analysis of Optical diffraction Response. Langmuir, 20: 3096-3106. DOI: 10.1021/la035555x

Sauer, M., A. Brecht, K. Charissé, M. Maier and M. Gerster et al., 1999. Interaction of chemically modified antisense oligonucleotides with sense DNA: A label-free interaction study with reflectometric interference spectroscopy. Anal. Chem., 71: 2850-2857. DOI: 10.1021/ac981057v

Soetedjo, H., 2011. Detections of water content changes in a nitrocellulose membrane based on polarized reflection spectroscopy. Sensors Transducers, 125: 100-106.

Soetedjo, H. and J. Räty, 2003. Reflectometric study of contaminant layer on a probe window. Applied Spectroscopy, 57: 915-919. PMID: 14661833

Soetedjo, H., M.F. Mora and C.D. Garcia, 2010. Optical properties of single-wall carbon nanotube films deposited on $\mathrm{Si} / \mathrm{SiO} 2$ wafers. Thin Solid Films, 518: $3954-3959 . \quad$ DOI: 10.1016/j.tsf.2010.02.037 